\title{
A KINETIC MODEL OF THE PLASMA FLOW AT THE MAGNETIC Z-PINCH AND THE PLASMOID STRUCTURE. PART 2
}

\author{
P. Kubes ${ }^{1}$, A. K. Prykarpatsky ${ }^{2}$, J. Zagrodzinski ${ }^{3}$, Y. A. Prykarpatsky ${ }^{3}$ \\ ${ }^{1}$ Dept. of Electrical Engeneering, Czech Technical University, \\ 2 Technicna Str., 16627, Praha-6, Czech Republic \\ ${ }^{2}$ Dept. of Applied Mathem. at the AGH, Krakow, 30-059, Poland \\ Dept. of Nonlinear Math. Analysis at the Institute for Applied Problems \\ of Mechanics and Math. of National Acad. Sci. of Ukraine, \\ 36 Naukova Str., Lviv, UA-290053, Ukraine \\ ${ }^{3}$ The Institute of Physics of the PAN, al. Lotnikow 32/46, Warsaw, 02-668, Poland
}

(Received May 20, 1997; received in final form May 6, 1998)

\begin{abstract}
In this article we will follow the approach developed in articles N. N. Bogoliubov, V. Hr. Samoilenko, Ukr. Fiz. Zh., 37, 147 (1992); J. Gibbon, Physica D 3, 503 (1981) using modern Lie-algebraic and symplectic geometry methods. It is devoted to the description of Boltzman-Vlasov type kinetic equations and some two-dimensional hydrodynamic Benney type flows associated with them. In our case of the cylindrical symmetry taking place at the interrupted magnetic z-pinch in plasma we used intensively the corresponding two-dimensionality of the plasma flow under consideration which made it possible to build a kinetic model of the plasmoid vortex structure with a conserved number of linkages of vortex lines. The latter can be used to explain the observed earlier stability of the plasmoid structure at the magnetic z-pinch.

Key words: kinetic model, plasma flow, vortex.
\end{abstract}

PACS numbers: $52.55 . \mathrm{Ez}$

\section{THE KINETIC MODEL OF THE PLASMA FLOW WITH CYLINDRICAL SYMMETRY}

Consider a multispecies collisionless plasma consisting of particles with the charges $e_{s}, s=\overline{1, N}$, moving in 3 -space $\mathbb{R}^{3}$, with the positions $\mathbf{x} \in \mathbb{R}^{3}$ and velocities $\mathbf{v} \in T^{*}\left(\mathbb{R}^{3}\right)$. We shall assume that the plasma densities $f^{s}(\mathbf{x}, \mathbf{v})$ as well as the electric and magnetic fields $\overline{\mathbf{E}}$ and $\overline{\mathbf{B}}$ are either periodic in $\mathbf{x} \in \mathbb{R}^{3}$, or have asymptotic decay sufficient to justify integration by parts. We shall also assume that $f^{s}, s \in \overline{1, N}$, decay to zero in the $\mathbf{v}$-variable as $v \rightarrow \infty$ at a sufficient rate that makes all subsequent integrals convergent. (Some weighted spaces may be appropriate here). The simplest Maxwell-Vlasov equations in $\mathbb{R}^{3}$ are:

$$
\frac{\partial f^{s}}{\partial t}+\left\langle\mathbf{v}, \frac{\partial f^{s}}{\partial \mathbf{x}}\right\rangle+\frac{e_{s}}{m_{s}}\left\langle\mathbf{E}+\mathbf{v} \times \mathbf{B}, \frac{\partial f^{s}}{\partial \mathbf{v}}\right\rangle=0
$$

$$
\frac{\partial \mathbf{B}}{\partial t}=-\operatorname{rot} \mathbf{E}
$$

$$
\operatorname{rot} \mathbf{B}=\mu_{0} \varepsilon_{0} \frac{\partial \mathbf{E}}{\partial t}+\mu_{0} \sum_{s=1}^{N} \int_{\mathbb{R}^{3}} d^{3} v \mathbf{v} f^{s}(\mathbf{x}, \mathbf{v}),
$$

$\operatorname{div} \mathbf{E}=\frac{1}{\varepsilon_{0}} \sum_{s=1}^{N} e_{s} \int_{\mathbb{R}^{3}} d^{3} v \mathbf{v} f^{s}(\mathbf{x}, \mathbf{v}), \quad \operatorname{div} \mathbf{B}=0$

where $\varepsilon_{0}$ and $\mu_{0}$ are the well known in electrodynamics constants, chosen further to be unity.

The equations of motion (1) are Hamiltonian with respect to the following Poisson bracket found from Lagrangian to Eulerian coordinates in $[3,4]$ for any $\gamma, \mu \in$ $D\left(T^{*}\left(\mathbb{R}^{3}\right)\right)$ :

$$
\begin{aligned}
\{\gamma, \mu\} & =\sum_{s=1}^{N} \int_{\mathbb{R}^{3}} d^{3} x \int_{\mathbb{R}^{3}} d^{3} v f^{s}\left[\left\{\frac{\delta \gamma}{\delta f^{s}}, \frac{\delta \mu}{\delta f^{s}}\right\}+\left(\left\langle\frac{\delta \gamma}{\delta \mathbf{E}}, \frac{\delta f}{\delta \mathbf{v}}\right\rangle \frac{\delta \mu}{\delta f^{s}}\right.\right. \\
& \left.\left.-\left\langle\frac{\delta \gamma}{\delta \mathbf{E}}, \frac{\delta f}{\delta \mathbf{v}}\right\rangle \frac{\delta \mu}{\delta f^{s}}\right)+\frac{e_{s}}{m_{s}} f^{s}\left\langle\mathbf{B}, \frac{\partial}{\partial \mathbf{v}}\left(\frac{\delta \gamma}{\delta f^{s}}\right) \times \frac{\partial}{\partial \mathbf{v}}\left(\frac{\delta \mu}{\delta f^{s}}\right)\right\rangle\right] \\
& +\int_{\mathbb{R}^{3}} d^{3} x\left(\left\langle\frac{\delta \gamma}{\delta \mathbf{E}}, \operatorname{rot} \frac{\delta \mu}{\delta \mathbf{B}}\right\rangle+\left\langle\frac{\delta \mu}{\delta \mathbf{E}}, \operatorname{rot} \frac{\delta \gamma}{\delta \mathbf{B}}\right\rangle\right) .
\end{aligned}
$$


The bracket (2) is a canonical one on the quotient space of the cotangent bundle of a principal fibre bundle by its structure group. Below we shall assume in more detail a one-component plasma flow over the constant charged background, possessing the cylindrical symmetry and giving rise to the vortex structure at the interrupted $\mathrm{z}$-pinch. To proceed with let us define a model distribution function $f=f\left(z, v_{z}\right)$ of plasma particles along $z$-axis under condition of the incompressibility. The resulting many-particle flow will model our plasma medium with the vortex structure if some reciprocity relationships are fulfilled upon the vortex boundary surface. Thus one can write down the following physically motivated simple equality:

$$
\int_{-\infty}^{u_{z}(r, z)} d v_{z} f\left(z, v_{z}\right)=\pi r^{2} n_{0}
$$

determining the vortex surface $r=R(z), z \in \mathbb{R}$, as follows:

$$
\int_{-\infty}^{u_{z}(R(z), z)} d v_{z} f\left(z, v_{z}\right)=\pi R^{2}(z) n_{0}
$$

In the above we denoted the hydrodynamic particle velocity as $\mathbf{v}=\left(u_{r}, u_{z}, 0\right)^{T} \in \mathbb{R}^{3}$, satisfying due to (3) the incompressibility condition $\operatorname{div} \mathbf{v}=0$, or

$$
\frac{\partial}{\partial r}\left(r u_{r}\right)+\frac{\partial}{\partial z}\left(r u_{z}\right)=0
$$

taking place for all $r \in \mathbb{R}_{+}, z \in \mathbb{R}$. Now we need to built proper Hamiltonian equations, governing the axisymmetric velocity vector $\mathbf{u} \in \mathbb{R}^{3}$. This can be done quite easily upon the reduced Poisson structure (2) in case when $N=1$ and upon the corresponding Hamiltonian function. The latter is constructed in terms of the moment function $a=\left\{a_{n} \in C^{\infty}(\mathbb{R} ; \mathbb{R}): n \in \mathbb{Z}_{+}\right\} \subset M_{\left(\mathbb{Z}_{+}\right)}$as follows:

$$
\begin{aligned}
H & =\frac{1}{2} \int_{\mathbb{R}} d z\left(a_{2}+\alpha^{2}+\alpha a_{0}\right. \\
& \left.+a_{1}\right)+\frac{1}{2} \int_{\mathbb{R}} d z \int_{0}^{R(z)}\left(|E|^{2}+|B|^{2}\right) \pi r d r,
\end{aligned}
$$

for some appropriate vortex fields $\alpha \in C^{\infty}(\mathbb{R}, \mathbb{R})$ and $\mathbf{E}, \mathbf{B} \in C^{\infty}\left(\mathbb{R}_{+} \times \mathbb{R}, \mathbb{R}\right)$, where by definition, for all $n \in \mathbb{Z}_{+}$

$$
a_{n}=\int_{\mathbb{R}} f\left(z, v_{z}\right) v_{z}^{n} d v_{z}
$$

Making use of the canonical transformations theory [7, 11] one can be convinced that the next representation follows from (3) and (4):

$$
a_{n}=\int_{0}^{R(z)} d r u_{z}^{n} \pi r n_{0}
$$

for all $n \in \mathbb{Z}_{+}$. The moment functionals (8) will be interpreted below magneto-hydrodynamically.

\section{THE MAGNETO-HYDRODYNAMIC MODEL OF THE PLASMA FLOW WITH CYLINDRICAL SYMMETRY}

Here we need to notice that electromagnetic field $\mathbf{E}$ and $\mathbf{B} \in C^{\infty}\left(\mathbb{R}_{+} \times \mathbb{R} ; \mathbb{R}\right)$ introduced above being axisymmetric, can be defined also as follows:

$$
\int_{-\infty}^{u_{z}+\frac{e}{m} A_{z}} d p_{z} \tilde{f}\left(z, p_{z}\right)=\pi r^{2} n_{0}
$$

where by the definition, for all $z, p_{z} \in \mathbb{R}$,

$$
\tilde{f}\left(z, p_{z}\right)=f\left(z, p_{z}-\frac{e}{m} A_{z}\right)
$$

- the distribution function on canonical phase space with the following evolution relation satisfied for all $t \in \mathbb{R}:$

$$
\frac{\partial \mathbf{A}}{\partial t}=-\mathbf{E}, \quad \operatorname{rot} \mathbf{A}=\mathbf{B}
$$

As a result of some simple calculations one obtains the following Hamiltonian equations of motion (in units when $2 \pi n_{0}=1$ ):

$$
\begin{aligned}
\frac{\partial u}{\partial t} & =-\frac{\partial u}{\partial z} u+\frac{\partial u}{\partial r} \frac{1}{r} \int_{0}^{r} r \frac{\partial u}{\partial z} d r-\frac{\partial u}{\partial z}-\frac{\partial \alpha}{\partial z} \\
& +\frac{e}{m}\left[\left(\frac{\partial A_{r}}{\partial z}-\frac{\partial A_{z}}{\partial r}\right) \frac{1}{r} \int_{0}^{r} r \frac{\partial u}{\partial z} d r\right]-\frac{\partial P}{\partial z} \\
\frac{\partial \alpha}{\partial t} & =-\frac{\partial \alpha}{\partial z}-\frac{\partial R}{\partial z}, \\
\frac{\partial R}{\partial t} & =-\frac{\partial R}{\partial z}-\frac{1}{R} \frac{\partial}{\partial z}\left(\int_{0}^{R(z)} r u d r\right)
\end{aligned}
$$

where we denoted by $P \in C^{\infty}\left(\mathbb{R}_{+} \times \mathbb{R} ; \mathbb{R}\right)$ the plasma pressure and put $u_{z}=u \in C^{\infty}\left(\mathbb{R}_{+} \times \mathbb{R}, \mathbb{R}\right)$. The Hamiltonian system (11) possesses the following important mathematical property: the algebraic curve

$$
\lambda=\frac{1}{2} \xi^{2}+\alpha+a(\xi)
$$

where for $\lambda, \xi \in \mathbb{C}$, 


$$
a(\xi)=\int_{0}^{R(z)} \frac{r d r}{\xi-u(r, z)-\frac{e}{m} A_{z}(r, z)},
$$

is invariant with respect to the flow (11). This property gives rise to a possibility of analysing the Hamiltonian system (11) by means of modern and powerful analytical methods developed during the past decades $[3-8,11]$. Especially, using the formula for the vorticity

$$
\boldsymbol{\omega}=\operatorname{rot} \mathbf{u}=\frac{\partial \mathbf{u}}{\partial r}+\frac{1}{r} \int_{0}^{r} r \frac{\partial^{2} \mathbf{u}}{\partial z^{2}} d r
$$

one can obtain the conditions making it possible for our system to have a nontrivial vortex solution. As can be derived from bracket (2), dynamical system (11) is Hamiltonian on a manifold $M_{(u, \alpha, R)} \subset C^{\infty}\left(\mathbb{R}_{+} \times \mathbb{R}_{\mathbb{R}^{2}} \times \mathbb{R}_{+}\right)$ with respect to a Poisson bracket reduced from the one before found in $[4,5]$ : for any $\gamma, \mu \in D\left(M_{(\bar{u} ; \bar{e}, \bar{b})}\right)$

$$
\begin{aligned}
\{\gamma, \mu\} & =\int_{\mathbb{R} \times \mathbb{R}_{+}} d z d r \pi r\left\langle\mathbf{u},\left[\left\langle\frac{\delta \gamma}{\delta \mathbf{u}}, \nabla\right\rangle \frac{\delta \mu}{\delta \mathbf{u}}-\left\langle\frac{\delta \mu}{\delta \mathbf{u}}, \nabla\right\rangle \frac{\delta \gamma}{\delta \mathbf{u}}\right]\right\rangle \\
& +\int_{\mathbb{R}_{+} \times \mathbb{R}} d z d r \pi r\left[\left\langle\frac{\delta \gamma}{\delta \mathbf{E}}, \operatorname{rot} \frac{\delta \mu}{\delta \mathbf{B}}\right\rangle-\left\langle\frac{\delta \mu}{\delta \mathbf{E}}, \operatorname{rot} \frac{\delta \gamma}{\delta \mathbf{B}}\right\rangle\right] \\
& +2 \int_{\mathbb{R}_{+} \times \mathbb{R}} d z d r \pi^{2} r\left[\left\langle\frac{\delta \gamma}{\delta \mathbf{u}}, \frac{\delta \mu}{\delta \mathbf{E}}\right\rangle-\left\langle\frac{\delta \mu}{\delta \mathbf{u}}, \frac{\delta \gamma}{\delta \mathbf{E}}\right\rangle+2 \pi \frac{e}{m}\left\langle\mathbf{B}, \frac{\delta \gamma}{\delta \mathbf{u}} \times \frac{\delta \mu}{\delta \mathbf{u}}\right\rangle\right],
\end{aligned}
$$

with both the incompressibility restriction div $\mathbf{u}=0$ and some boundary conditions [6, 11] involved upon the vortex structure. Since the vortex surface $\sigma \subset \mathbb{R}^{3}$ is defined by the free boundary equation $r=R(z)$ for $z \in \mathbb{R}$ in the cylindrical coordinates because of assumed axisymmetry of the plasma motion, we will further consider the surface $\sigma$ to be compact and diffeomorphic to the boundary of some axisymmetric reference region $\Omega_{\sigma}$. The Hamiltonian function (6) is subject to a generalization taking into account boundary terms on the vortex surface $\sigma: H \rightarrow H_{\sigma}$, where

$$
H_{\sigma}=\frac{1}{2} \int R d z\left(a_{2}+\alpha^{2}+\alpha a_{0}+a_{1}\right)+\frac{1}{2} \int_{\mathbb{R}} d z \int_{0}^{R(z)}\left(|E|^{2}+|B|^{2}\right) \pi r d r+\tau \int_{\sigma} d S
$$

where $\tau \in \mathbb{R}_{+}$is the vortex surface tension, $d S$ is an infinitesimal area element of the surface $\sigma$. Upon the vortex surface $\sigma$ the following boundary relationships are to be satisfied:

$$
\frac{\partial \sigma}{\partial t}=\left\langle\mathbf{u}, \mathbf{n}_{\sigma}\right\rangle,\left.\quad P\right|_{\sigma}=\tau k
$$

where $\mathbf{n}_{\sigma} \in C^{\infty}\left(\mathbb{R} ; \mathbb{R}^{3}\right)$ is the unit outward normal to the surface $\sigma, k \in C^{\infty}(\mathbb{R} ; \mathbb{R})$ is the mean curvature of the vortex surface $\sigma, P \in C^{\infty}(\mathbb{R}+\times \mathbb{R} ; \mathbb{R})$ is the above introduced plasma pressure. Just as stated in [13], the resulting still unreduced upon the submanifold div $\mathbf{u}=0$ Poisson bracket can be written as follows: for any $\gamma, \mu \in D\left(M_{(\bar{u} ; \bar{e}, \bar{b})}\right)$

$$
\{\gamma, \mu\}_{\sigma}=\{\gamma, \mu\}+\int_{\sigma} d S\left(\frac{\delta \gamma}{\delta \sigma} \frac{\delta \mu}{\delta \varphi}-\frac{\delta \gamma}{\delta \varphi} \frac{\delta \mu}{\delta \sigma}\right)
$$

where $\varphi \in C^{\infty}\left(\mathbb{R}_{+} \times \mathbb{R} ; \mathbb{R}\right)$ is the velocity potential uniquely obtained from the following decomposition:

$$
\mathbf{u}=\mathbf{w}+\nabla \varphi, \triangle \varphi=0,\left.\frac{\delta \varphi}{\delta n}\right|_{\sigma}=\left\langle\mathbf{u}, \mathbf{n}_{\sigma}\right\rangle
$$

The velocity component $\mathbf{w} \in C^{\infty}\left(\mathbb{R}_{+} \times \mathbb{R} ; \mathbb{R}^{3}\right)$ is obviously divergent free and tangent to the vortex surface $\sigma$. As a result of the above decomposition the variational derivative $\left.\frac{\partial \gamma}{\partial \varphi}\right|_{\sigma}$ for any functional $\gamma \in D\left(M_{(u)}\right)$ is defined by the rule:

$$
\left.\frac{\delta \gamma}{\delta \varphi}\right|_{\sigma}=\left\langle\frac{\delta \gamma}{\delta \mathbf{u}}, \mathbf{n}_{\sigma}\right\rangle
$$

The corresponding derivative $\frac{\delta \gamma}{\delta \varphi}$ is slightly more involved. A variation $\delta \sigma$ of the vortex surface $\sigma$ is identified with a function on $\sigma$; it represents an infinitesimal variation of $\sigma$ in its normal direction $\mathbf{n}_{\sigma}$. By the incompressibility assumption, $\delta \sigma$ has zero integral on $\sigma$, a condition dual to the additive constant ambiguity in $\varphi$. Smoothly extend the velocity $\mathbf{u}$ so it is defined in a neighborhood of $\sigma$; thus, holding $\mathbf{u}$ constant while varying $\sigma$ makes sense. Then one can set 


$$
\delta \gamma=\int_{\sigma} \frac{\delta \gamma}{\delta \sigma} \delta \sigma d S
$$

determining $\frac{\delta \gamma}{\delta \sigma}$ up to an additive constant. It is easily checked that $\frac{\delta \gamma}{\delta \sigma}$ is independent on the way the velocity $\mathbf{u}$ is extended as long as the functional $\gamma$ is $C^{1}$ as $\mathbf{u}$ varies in the $C^{1}$-topology. Note here also that the plasma pressure $P$ in (17) can be determined as the solution to the Dirichlet problem:

$$
\left.P\right|_{\sigma}=\tau k, \triangle P=-\operatorname{div}(\langle\mathbf{u}, \nabla\rangle \mathbf{u}) .
$$

After an appropriate reduction of the Poisson bracket (18) upon the invariant submanifold div $\mathbf{u}=0$ the Poisson bracket on the manifold $M_{(u, a, R ; E, B)}$ coincides with the reduced Poisson bracket (2) via the transformation (3) to the velocity variables. The above mentioned reduction procedures are of quite complicated form, so for the moment they are omitted from the present treatment of the problem.

\section{THE VORTEX AND HELICITY STRUCTURE ANALYSIS}

To proceed further with the study of the peculiar vortex type and helicity solutions to the vortex modeling hydrodynamic system (11), let us first discuss its so called Riemannian invariants [7]. To compute them in explicit form, we shall recall here that the algebraic function (12) is invariant with respect to the evolution (11).

The corresponding winding points of curve (12) are determined by the condition $\frac{d \lambda}{d \xi}=0, \xi \in C$. Since $\lambda \in \mathbb{C}$ is the invariant "spectral" parameter, the critical points $\xi_{j}=\xi_{j}(z, r, t), j=\overline{1, N_{\lambda}}$, generate on the $(z, r)$-plane the following set of characteristics

$$
\frac{d \lambda_{j}}{d t}=\left.\frac{\partial \lambda}{\partial z}\right|_{\lambda=\lambda_{j}} u-\left.\frac{\partial \lambda}{\partial r}\right|_{\lambda=\lambda_{j}}\left(\frac{1}{r} \int_{0}^{r} d r r \frac{\partial u}{\partial z}\right)
$$

where $\lambda_{j}=\lambda\left(\xi_{j}\right), \lambda\left(\xi_{j}\right)=0$ for all $j=\overline{1, N_{\lambda}}$. Having obtained the information about the motion in the $(z, r)-$ plane of the quasi-invariants $\lambda_{j} \in D\left(M_{(u, R ; E, B)}\right) j=$ $\overline{1, N_{\lambda}}$, one can build explicitly some helicity conserved functionals sufficing the wanted existence of the vortex structure in the plasma. Assuming the superfluid approximation of the plasma motion, that is putting

$$
\begin{aligned}
& \frac{\partial \mathbf{A}}{\partial t}=\mathbf{u} \times \mathbf{B}, \operatorname{rot} \mathbf{B}=\mu_{0}\left(\mathbf{j}+\varepsilon_{0} \frac{\partial \mathbf{E}}{\partial t}\right), \\
& \frac{\partial \mathbf{B}}{\partial t}=-\operatorname{rot} \mathbf{E}, \\
& j=e n_{0} \mathbf{u}, n_{0} m \frac{d \mathbf{u}}{d t}=-\nabla p+\mathbf{j} \times \mathbf{B}, \operatorname{rot} \mathbf{A}=\mathbf{B},
\end{aligned}
$$

one can prove [8] that the following helicity functional is conserved, i.e. for all $t \in \mathbb{R}$

$$
\frac{\partial \Lambda}{\partial t}=0, \Lambda=\int_{\Omega_{\sigma}} d r \pi r d z\langle\mathbf{q}, \operatorname{rot} \mathbf{q}\rangle
$$

where $\mathbf{q}=\mathbf{u}+\frac{e}{m} \mathbf{A}$. The result (25) generalizes evidently the well known Kelvin theorem on the helicity, representing the number of linkages of the vortex lines in the domain $\Omega_{\sigma}$ with the surface $\sigma$ [8].

An appropriate nonlinear analysis of vector fields (23) concerned with the algebraic curve (12) show that there exist zeroes $\lambda_{j k}, k=\overline{1, N_{\lambda}}$, creating some linked closed lines, thereby suggesting the existence of the vortex structure in the plasma flow under consideration. A much more detailed analysis of this possibility created by the isospectral representation (12), (13) will be delivered in the subsequent Part 3 under preparation.

\section{ACKNOWLEDGMENTS}

The authors are thankful to Dr. J. Stakhira for helpful discussions, Dr. J. Tavantzis and Dr. D. Blackmore for sending their reprints on the vortex structures. This work was done while one of the authors (A.P.) benefited from a support by the Czech Technical University, Dept. of Electrical Engineering which made research visits possible. This research has been supported by grants GACR No.202-95-0178 "Stable Structures in Magnetic Pinches" and No. 202-97-0487 " $X$-ray Source on the Magnetic Pinch Principle". The authors are indebted to the anonymous referee whose remarks and comments made it possible to improve the presentation in the articles.
[1] N. N. Bogoliubov, V. Hr. Samoilenko, Ukr. Fiz. Zh., 37, 147 (1992).

[2] J. Gibbon, Physica D 3, 503 (1981).

[3] J. Marsden, A. Weinstein, Physica D 4, 394 (1982).

[4] J. Marsden, T. Ratiu, A. Weinstein, Trans. Am. Soc. Met. 28, 147 (1984).

[5] B. A. Kupershmidt, T. Ratiu, Commun. Math. Phys. 90,
235 (1983).

[6] D. Levis, J. Marsden, R. Montgomery, T. Ratiu, Physica D 18, 391 (1986)

[7] G. B. Whitham, Linear and nonlinear waves (John Wiley\& Sons, New York, 1974).

[8] D. Holm, B. A. Kupershmidt, Phys. Rev. A 36, 3947 (1987). 
[9] H. K. Moflat, Magnetic field generation in electrically conducting fluids (Cambridge University Press, Cambridge, England, 1978).
[10] D. Blackmore, Acta Mechanica 102, 91 (1994).

[11] G. A. Goldin, R. Menikoff, D. H. Sharp, Phys. Rev. Lett. 58, $2162(1987)$.

\title{
КІНЕТИЧНА МОДЕЛЬ ПЛ АЗМОВОГО ПОТОКУ ПРИ МАГНЕТНОМУ Z-ППНЧІ ТА ЙОГО ПЛ АЗМӦ̈ДНА СТРУКТУРА. ЧАСТИНА 2
}

\author{
П. Кубеш ${ }^{1}$, А. К. Прикарпатський ${ }^{2}$, Є. Загродзінскі ${ }^{3}$, Я. А. Прикарпатський ${ }^{3}$ \\ ${ }^{1}$ Чесъкий технічний університет, факультет електричнӧ̈ інженерій, \\ вул. Технічна, 2, 16627, Прага-6, Чесъка республіка \\ E-mail: kubes@feld.cvut.cz \\ ${ }^{2}$ Факультет прикладнойматематики АГХ, Краків, Зо-059, Польща, \\ відділ нелінійного математичного аналізу ІППММ НАН Украйни, \\ вул. Наукова, 3б, Львів, 290053, Украйна \\ E-mail:prykan@cybergal.com \\ ${ }^{3}$ Iнститут фізики ПАН, ал. Льотнікуб, 32/46, Варшаба, 02-668, Польща \\ E-mail: yarchyk@mailcity.com
}

У цій роботі ми продовжуємо розвинуте в статтях N. N. Bogoliubov, V. Hr. Samoilenko, Ukr. Fiz. Zh., 37, 147 (1992); J. Gibbon, Physica D 3, 503 (1981) дослідження. Воно присвячене описові кінетичних рівнянь Больцмана-Власова й асоційованих з ними двовимірних гідродинамічних потоків Бенні, з використанням сучасних Лі-алгебраїчних методів та методів симплектичної геометрії. У випадку циліндричної симетрії у плазмі має місце перерваний z-пінч, тому використання відповідного двовимірного плазмового потоку дало змогу побудувати кінетичну модель плазмоїдної вихрової структу ри зі сталою кількістю зв'язних вихрових ліній. Це може бути використано для пояснення стабільности плазмоїдної структури при магнетному zпінчі. 